\title{
Expression of angiopoietin-TIE system components in angiosarcoma
}

\author{
Darya Buehler ${ }^{1}$, Patrick Rush ${ }^{1}$, Jason R Hasenstein ${ }^{2}$, Stephanie R Rice ${ }^{2}$, \\ Gholam Reza Hafez ${ }^{1}$, B Jack Longley ${ }^{3}$ and Kevin R Kozak ${ }^{2}$
}

${ }^{1}$ Department of Pathology, University of Wisconsin School of Medicine and Public Health, Madison, WI, USA; ${ }^{2}$ Department of Human Oncology, University of Wisconsin School of Medicine and Public Health, Madison, WI, USA and ${ }^{3}$ Department of Dermatology, University of Wisconsin School of Medicine and Public Health, Madison, WI, USA

\begin{abstract}
Angiosarcoma is an aggressive malignancy of endothelial differentiation. Potential roles of the endothelial angiopoietin-tunica interna endothelial cell kinase (ANGPT-TIE) system in angiosarcoma diagnosis, pathogenesis, prognosis and treatment are undefined. To examine the expression and prognostic significance of angiopoietin-1, angiopoietin-2, TIE1 and TEK (TIE2) proteins in angiosarcoma, we immunohistochemically evaluated clinically annotated human angiosarcoma samples. Correlations of protein expression with overall survival and pathological features were explored. The cohort included 51 patients diagnosed with angiosarcoma at the age of 30-86 years (median 67 ). The 5-year overall survival was $45 \%$ with a median of 26 months. Moderate to strong expression of angiopoietin-1, TIE1 and TEK (TIE2) was identified in the majority of angiosarcomas and moderate to strong expression of angiopoietin-2 was observed in $42 \%$ of angiosarcomas. Increased angiopoietin-1 expression correlated with improved survival. Non-significant trends toward longer survival were also observed with increased TIE1 and TEK (TIE2) expression. Increased expression of angiopoietin-2, TIE1 and TEK (TIE2) was associated with vasoformative architecture. No differences in expression of these proteins were observed when patients were segregated by age, gender, presence or absence of metastases at diagnosis, primary tumor location, radiation association or the presence of necrosis. We conclude that components of the ANGPT-TIE system are commonly expressed in angiosarcomas. Reduced expression of these proteins is associated with nonvasoformative and clinically more aggressive lesions.

Modern Pathology (2013) 26, 1032-1040; doi:10.1038/modpathol.2013.43; published online 5 April 2013
\end{abstract}

Keywords: angiosarcoma; angiopoietin; sarcoma; TEK; TIE

Angiosarcoma is an aggressive soft tissue sarcoma of endothelial differentiation. Current therapies are woefully inadequate. Patients presenting with localized disease have a 5-year overall survival of $<60 \%$. Those presenting with metastatic disease fare far worse with median survival generally $<1$ year. ${ }^{1-5}$ The poor survival of patients with metastatic disease is largely attributable to limited systemic therapy options. Reported response rates with conventional cytotoxic chemotherapy are highly variable, however, they are generally similar to the disappointing response rates seen in other soft tissue sarcomas (ie, $20-30 \%)^{6}$

Correspondence: Professor KR Kozak, MD, PhD, Department of Human Oncology, University of Wisconsin School of Medicine and Public Health, Wisconsin Institute for Medical Research, 1111 Highland Avenue, Madison, WI 53705, USA.

E-mail: kozak@humonc.wisc.edu

Received 12 September 2012; revised 7 January 2013; accepted 8 January 2013; published online 5 April 2013
Given the vascular differentiation of angiosarcomas, recent interest has turned to therapies that target endothelium-restricted signaling pathways. Vascular endothelial growth factors (VEGFs) and their receptors are expressed in angiosarcoma. ${ }^{7-14}$ Moreover, activating mutations in KDR (VEGFR-2), the major pro-angiogenic VEGF receptor, have been reported in a small subset of angiosarcomas. ${ }^{15}$ Consequently, the therapeutic use of anti-VEGF agents has been explored in this disease. The pan-VEGF receptor tyrosine kinase inhibitor, sorafenib, has modest single-agent activity in angiosarcoma. ${ }^{16,17}$ Similarly, the humanized antiVEGF monoclonal antibody, bevacizumab, elicits objective responses in a minority of angiosarcoma patients. ${ }^{18,19}$

The ANGPT-TIE pathway is largely confined to vasculature and consists of two tyrosine kinase receptors, TIE1 and TEK (TIE2), and three corresponding ligands, angiopoietins-1, -2 and -4 . Although considerable context-dependent altera- 
tions in function have been observed, generally, angiopoietin-1 acts as a TEK (TIE2) receptor agonist and angiopoietin-2 as a TEK (TIE2) receptor antagonist. The TIE1 receptor has no known ligand and appears to function primarily as a TEK (TIE2) receptor antagonist through interference of angiopoietin-1 receptor interaction. ${ }^{20}$ Angiopoietin-1 has a key role in maintaining the integrity of existing vessels and enhances endothelial cell survival, proliferation and migration in some settings. ${ }^{21-25}$ Angiopoietin-2 appears to have a critical role in vascular remodeling and angiogenesis. ${ }^{26}$ The functions of the more recently described ligand, angiopoietin-4, are less well understood and its expression is largely limited to the lung. ${ }^{27}$

Little information is available regarding the expression of ANGPT-TIE pathway components in human angiosarcoma samples. ${ }^{12,15,28}$ Recently, after identifying TEK (TIE2) expression in 11 human angiosarcomas, we reported that TEK (TIE2) inhibition delayed angiosarcoma growth in two murine models of the disease. ${ }^{29}$ To examine potential roles for ANGPT-TIE pathway components in angiosarcoma diagnosis, pathogenesis, prognosis and treatment, we assessed the expression of angiopoietin-1, angiopoietin-2, TIE1 and TEK (TIE2) by immunohistochemistry in 51 clinically annotated human angiosarcoma samples.

\section{Materials and methods}

\section{Patients and Angiosarcoma Specimens}

The study was approved by the Institutional Review Board. Patients were identified using a surgical pathology database that spanned the years 1987-2012. Candidate paraffin blocks were collected and the angiosarcoma diagnosis was confirmed by a pathologist with specific interest in soft tissue and skin tumors (GRH and BJL). The presence or absence of necrosis was noted. Tumor architectural pattern was assessed according to Shon et al $\mathrm{l}^{30}$ as follows: vasoformative ( $>75 \%$ of tumor forming vascular channels with identifiable lumina), nonvasoformative $(>75 \%$ of tumor demonstrating architecturally solid epithelioid or spindle cell morphology without vascular channels) or mixed. Patient records were accessed for age at diagnosis, sex, disease distribution at diagnosis, date of last follow-up or death, primary site and tumor size. Clinical notes and radiation records were reviewed. Angiosarcomas were deemed radiation associated if they occurred within or adjacent to prior radiation fields.

\section{Tissue Microarray Construction}

Fifty-one paraffin blocks from angiosarcoma surgical specimens were identified. No specimen was obtained following neoadjuvant radiation or chemotherapy. After confirmation of the diagnosis, the most representative viable areas of the formalinfixed paraffin-embedded angiosarcomas were formatted into a $4.5 \times 2 \times 1 \mathrm{~cm}^{3}$ recipient tissue microarray in triplicate $1.0 \mathrm{~mm}$ cores using the MTA-1 manual tissue microarrayer (Beecher Instruments, Sun Prairie, WI, USA). The 11 angiosarcoma cases previously reported to express TEK (TIE2) in wholemount tissue sections were included in the tissue microarray. ${ }^{29}$ In five cases (two breast, two head and neck, and one Stewart-Treves), limited tumor tissue necessitated use of only two tissue cores. Sections were cut $(5 \mu \mathrm{m})$ and standard H\&E and CD31 stained slides were examined to verify the presence of viable angiosarcoma.

\section{Immunohistochemistry}

Tissue sections $(5 \mu \mathrm{m})$ were cut using traditional water bath technique and dried overnight at room temperature. Slides were deparaffinized in subsequent xylene and ethanol incubations followed by heat induced epitope retrieval using the Lab Vision PT module (Thermo Fisher Scientific, Fremont, CA, USA) with Lab Vision citrate buffer $\mathrm{pH} 6.0$ at $98^{\circ} \mathrm{C}$ for $20 \mathrm{~min}$ without boiling. All staining was performed at room temperature using the Lab Vision 360 automated staining system. Biocare Medical (Concord, CA, USA) reagents were used except where noted. Endogenous peroxidase was blocked for $5 \mathrm{~min}$ with Peroxidazed 1. Nonspecific protein binding was inhibited by a 10-min (angiopoietin-2, TIE1, TEK (TIE2)) or $30 \mathrm{~min}$ (angiopoietin-1) block with Sniper. For angiopoietin-1, nonspecific avidin binding was blocked using the Avidin Biotin kit, incubating $15 \mathrm{~min}$ for each reagent. Primary antibodies were diluted with DaVinci Green Antibody Diluent (angiopoietin-2, TIE1, TEK (TIE2)) or Renaissance background reducing diluent (angiopoietin-1). Slides were incubated with primary antibodies for $60 \mathrm{~min}$ as follows: angiopoietin-1 (goat anti-angiopoietin-1 IgG, 1:50, R\&D Systems, Minneapolis, MN, USA), angiopoietin-2 (mouse anti-angiopoietin-2 IgG, 1:50, Santa Cruz Biotechnology, Santa Cruz, CA, USA), TIE1 (mouse antiTIE1 IgG, 1:300, R\&D Systems), TEK (goat anti-TEK IgG, 1:50, R\&D Systems). For angiopoietin-2 and TIE1 stains, slides were subsequently incubated for 20 min with probe and HRP-polymer from mouse HRP-Polymer kit. For TEK (TIE2), similar incubations were performed with the goat HRP-polymer kit. For angiopoietin-1, primary incubation was followed by incubation for $15 \mathrm{~min}$ with biotinylated swine anti-goat IgG (1:50, Invitrogen, Carlsbad, CA, USA) followed by a 15-min streptavidin-HRP treatment. Betazoid DAB and Mayer's hematoxylin were each incubated for $1 \mathrm{~min}$. Slides were washed with Tris-buffered saline-Tween between steps. For CD31 immunolabeling, the heat-induced epitope retrieval was performed using Dako's FLEX Target Retrieval 
Solution, Low $\mathrm{pH}$ (pH 6.0) for $45 \mathrm{~min}$ in a decloaker. Endogenous peroxidase was blocked with $300 \mu \mathrm{l}$ of Dako's peroxidase blocking reagent for $5 \mathrm{~min}$. Slides were then incubated on Dako Autostainer with Dako FLEX ready-to-use CD31 antibody (mouse anti-CD31 IgG, Dako, Carpinteria, CA, USA) at room temperature for $20 \mathrm{~min}$ followed by a Mouse LINKER for $15 \mathrm{~min}$ and Dako's FLEX detection reagent for $15 \mathrm{~min}$. DAB reagent was then applied for $5 \mathrm{~min}$ followed by hematoxylin counterstain. Immunoreactivity was scored by a pathologist blinded to patient outcomes using conventional brightfield microscopy. Cytoplasmic and membranous staining was interpreted as follows: 0, no staining; 1, weak staining; 2, moderate staining; 3, strong staining. For each patient, a single immunohistochemical score was generated by averaging the scores of all represented cores.

\section{Statistical Analysis}

Five-year overall survival comparisons were performed using the Kaplan-Meier method. Statistical significance was calculated by log-rank test. Survival curves were generated using GraphPad Prism software (Version 5.0, La Jolla, CA, USA). Fisher's exact test was used to assess differences in protein expression between patient subgroups.

\section{Results}

\section{Patient and Tumor Characteristics}

Patient and tumor characteristics are presented in Table 1. The cohort consisted of 30 females and 21 males, with a median age of 67 years at diagnosis (range, 30-86 years). The majority (69\%) of patients presented with localized disease. Sixteen patients $(31 \%)$ had distant metastases at diagnosis. Nearly $80 \%$ of patients presented with visceral/deep soft tissue, head and neck or cutaneous primaries. Visceral/deep soft tissue sites included liver $(N=3)$, bone $(N=3)$, bladder $(N=3)$, lung $(N=2)$, pelvic soft tissue $(N=2)$, bowel $(N=2)$, and one each of the following: spleen, ovary, seminal vesicle, adrenal and aorta. Twelve cases (24\%) were considered to be radiation associated, with the majority $(7 / 12,58 \%)$ being related to radiation therapy for breast cancer. Two-thirds of primary lesions were $5 \mathrm{~cm}$ or less in greatest dimension. The most common histological pattern was vasoformative; $43 \%$ of tumors displayed necrosis. Considering the entire cohort, 5 -year overall survival was $45 \%$ with a median of 26 months.

\section{Angiopoietin-1, Angiopoietin-2, TIE1 and TEK (TIE2) Expression}

Angiopoietin-1 immunoreactivity was found in the vast majority of angiosarcomas; $3(6 \%), 4(8 \%)$, $26(51 \%)$ and $18(35 \%)$ of 51 lesions displayed no
Table 1 Patient and tumor characteristics

$\mathrm{N}(\%$ total $)$

Patient characteristics

Age, median (range, years)

67 (range, 30-86)

Gender

$21(41 \%)$

Female

$30(59 \%)$

Localized disease

Metastatic disease

$35(69 \%)$

$16(31 \%)$

Vital status

Alive

$19(37 \%)$

Deceased

$32(63 \%)$

Tumor characteristics

Primary site

Visceral/deep soft tissue

Head and neck/cutaneous

Breast

Stewart-Treves

$20(39 \%)$

$20(39 \%)$

$8(16 \%)$

$3(6 \%)$

Radiation associated

Breast

Bladder

Liver

Sternum

Scalp

$12(24 \%)$

$7(58 \%)$

$2(17 \%)$

$1(8 \%)$

$1(8 \%)$

$1(8 \%)$

Primary tumor size:

Total available

$\leqslant 5 \mathrm{~cm}$

45

$>5 \mathrm{~cm}$

$30(67 \%)$

$15(33 \%)$

Histological pattern

Vasoformative

Epithelioid

Spindle mixed necrosis

Mixed

$30(59 \%)$

$6(12 \%)$

$2(4 \%)$

$13(25 \%)$

Necrosis

Absent $29(57 \%)$

Present

$22(43 \%)$

Total

51

$(0+)$, weak $(1+)$, moderate $(2+)$ and strong $(3+)$ staining, respectively (Figure 1, rows 1 and 2). Among the seven specimens with no-to-weak angiopoietin-1 immunoreactivity, three demonstrated weak, three demonstrated moderate and one demonstrated strong TEK (TIE2) expression (data not shown). Angiopoietin-2 staining was more heterogeneous with $19(38 \%), 10(20 \%), 10(20 \%)$ and 11 $(22 \%)$ of 50 specimens showing no, weak, moderate and strong immunoreactivity, respectively (Figure 1, rows 3 and 4). A single specimen, represented by two cores, was lost from the tissue microarray during the angiopoietin-2 immunostaining procedure. TIE1 and TEK (TIE2) immunoreactivity largely paralleled angiopoietin-1 staining with the majority of samples displaying moderate to strong expression of these receptors. For TIE1, absent, weak, moderate and strong staining was observed in $5(10 \%), 11$ $(22 \%), 15(29 \%)$ and $20(39 \%)$ of 51 angiosarcomas, 


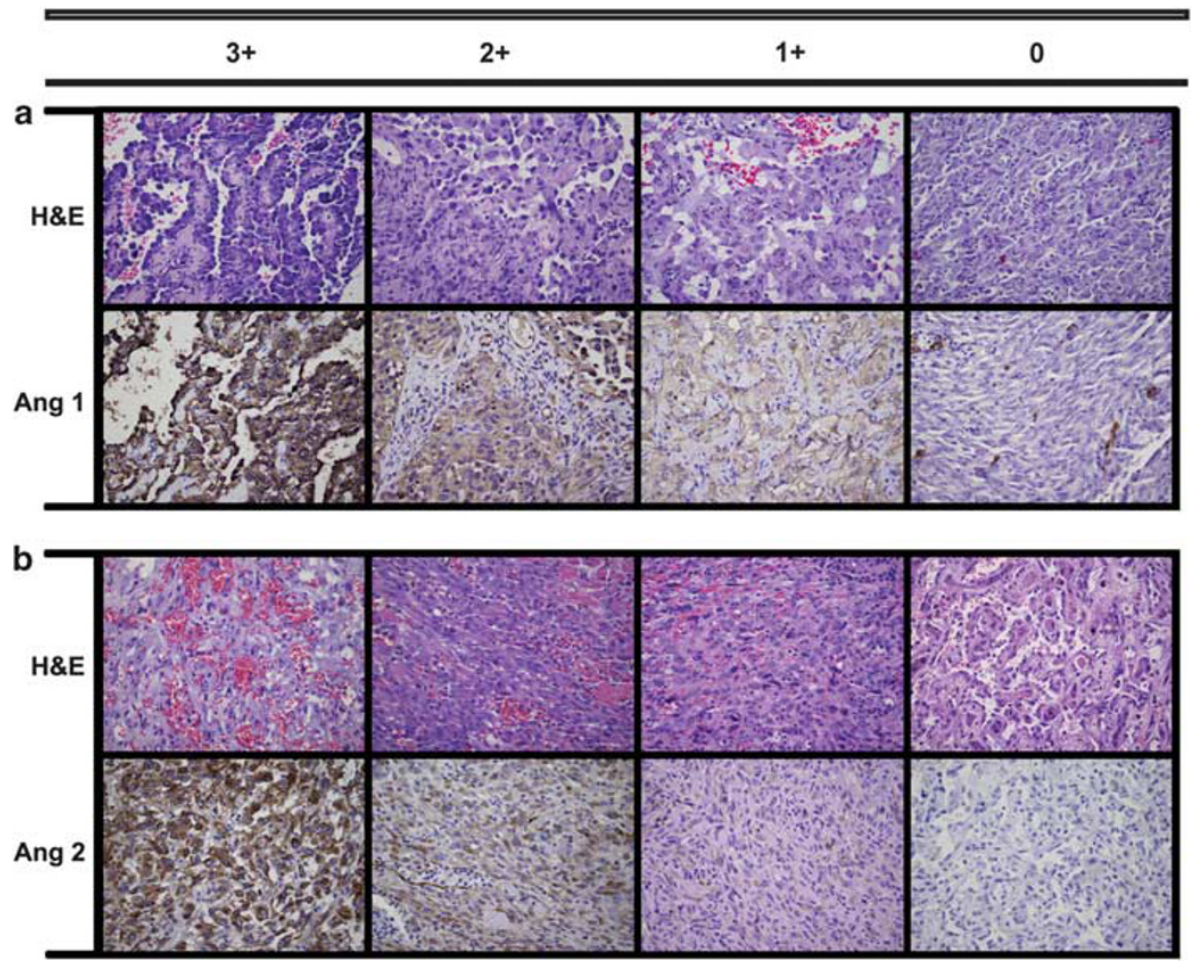

Figure 1 Representative images displaying the spectrum of angiopoietin-1 (a) and angiopoietin-2 (b) immunoreactivity in human angiosarcomas. Corresponding H\&E stained images are also displayed (original magnification $\times 400$ ).

respectively (Figure 2, rows 1 and 2). TEK (TIE2) immunoreactivity was not observed in only one sample. In the remaining 50 specimens, TEK (TIE2) expression was found to be weak, moderate and strong in $9(18 \%), 20(39 \%)$ and $21(41 \%)$ cases, respectively (Figure 2, rows 3 and 4).

\section{ANGPT-TIE System Proteins and Overall Survival}

Correlations between angiopoietin-1, angiopoietin2, TIE1 and TEK (TIE2) immunostaining and overall survival are shown in Figure 3 and Table 2 . Generally, expression of angiopoietin-1, TIE1 and TEK (TIE2) was associated with improved overall survival. However, this reached statistical significance only with angiopoietin-1. Among the seven patients with no or low angiopoietin-1 immunoreactivity, none survived for 3 years. In contrast, among patients with moderate to strong expression of angiopoietin-1, median overall survival approached 4 years.

\section{Correlations of Angiopoietin and TIE Expression With Patient and Tumor Characteristics}

To identify potential correlations between angiopoietin-1, angiopoietin-2, TIE1 and TEK (TIE2) immunoreactivity and angiosarcoma patient and tumor characteristics, the samples were segregated by clinical and pathological variables (Table 3). Expression patterns did not differ by age, gender, metastatic disease at presentation, radiation association or tumor size. Increased TIE receptor immunoreactivity was strongly associated with vasoformative histology. Similarly, increased angiopoietin staining was observed in vasoformative angiosarcomas; however, this did not reach statistical significance for angiopoietin-1. Breast angiosarcomas and the three angiosarcomas arising in the background of chronic lymphedema (Stewart-Treves syndrome) were associated with near-universal moderate-tostrong immunoreactivity for angiopoietin-1, TIE1 and TEK (TIE2). However, this did not differ in a statistically significant way when compared with more common primary sites. Notably, all breast angiosarcomas and the three angiosarcomas associated with Stewart-Treves syndrome displayed vasoformative architecture.

\section{Discussion}

The ANGPT-TIE system has an essential role in developmental angiogenesis. ${ }^{31-36}$ Additionally, this complex vascular signaling architecture has been implicated in tumor progression. Elevated angiopoietin-1 expression has been correlated with improved disease-free survival in head and neck squamous cell carcinomas although it appears to have little prognostic significance in most solid tumors. ${ }^{37-40}$ In contrast, overexpression of angiopoietin-2 and elevated angiopoietin-2/ angiopoietin-1 ratios correlate with worse survival 

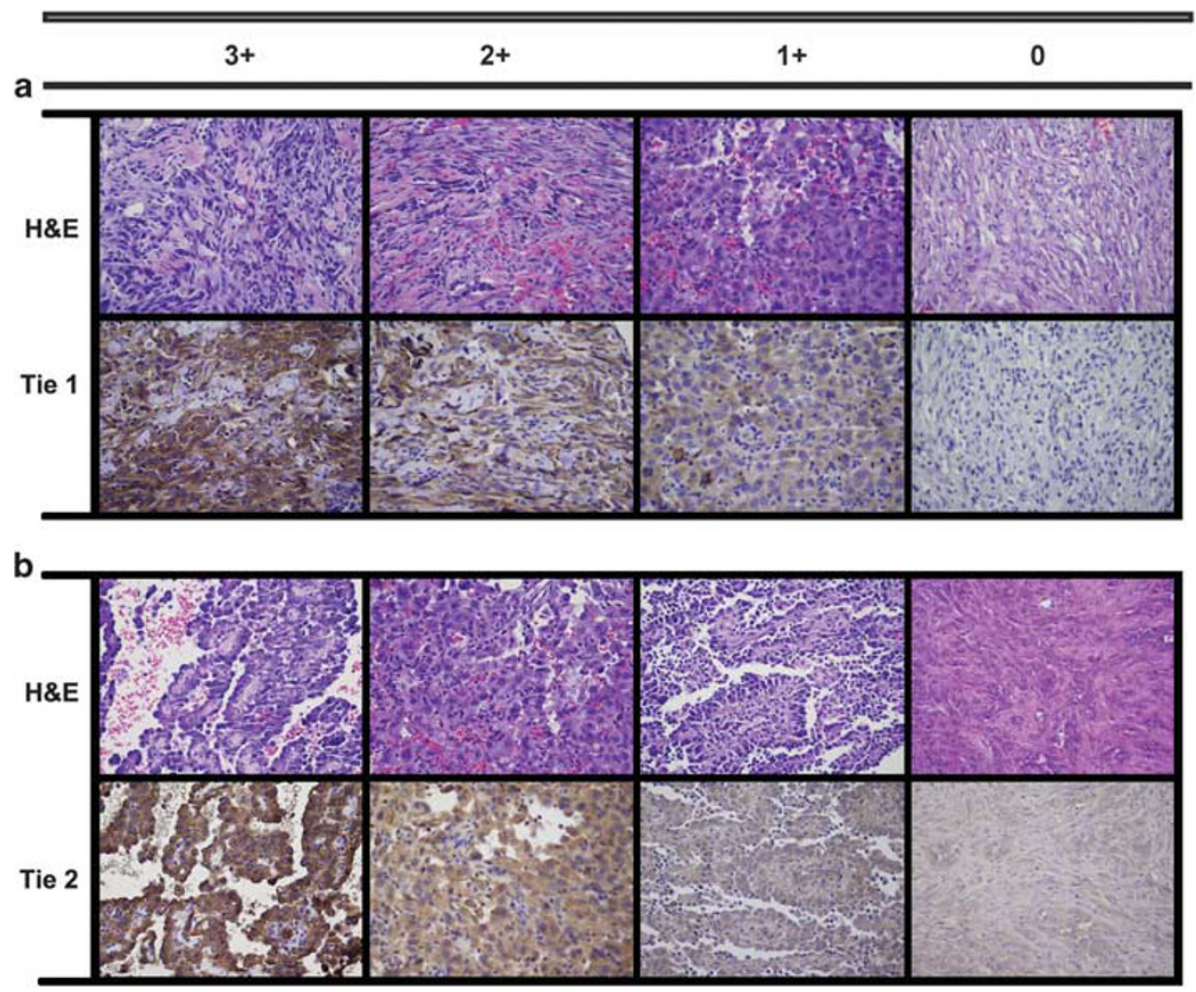

Figure 2 Representative images displaying the spectrum of TIE1 (a) and TEK (TIE2) (b) immunoreactivity in human angiosarcomas. Corresponding H\&E stained images are also displayed (original magnification $\times 400$ ).
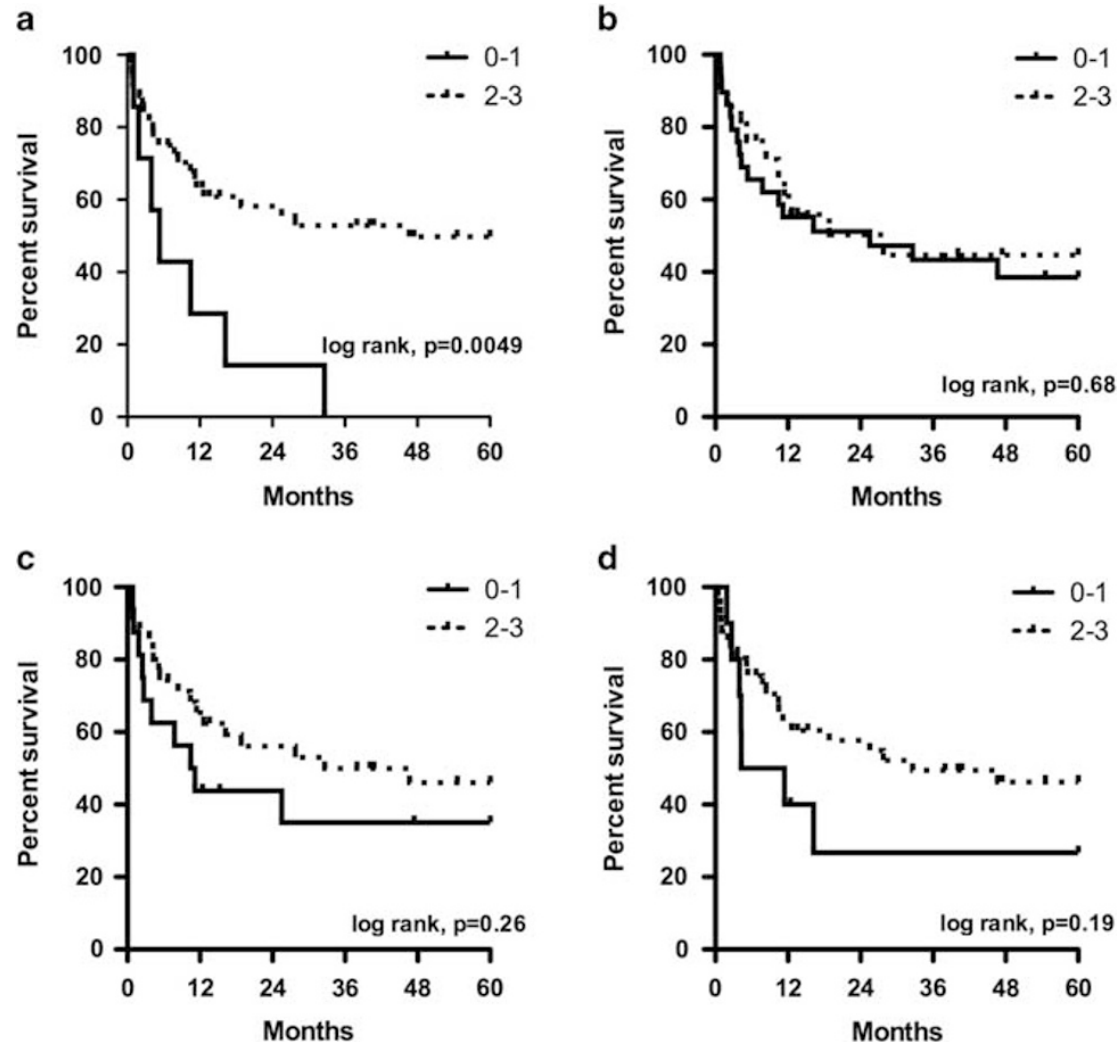

Figure 3 Overall survival of angiosarcoma patients segregated by tumor expression of angiopoietin-1 (a), angiopoietin-2 (b), TIE1 (c) and TEK (TIE2) (d). 
Table 2 Overall survival based on angiopoietin and TIE expression

\begin{tabular}{|c|c|c|c|c|c|c|c|c|}
\hline & \multicolumn{2}{|c|}{ Angiopoietin-1 } & \multicolumn{2}{|c|}{ Angiopoietin-2 } & \multicolumn{2}{|c|}{ TIE1 } & \multicolumn{2}{|c|}{ TEK (TIE2) } \\
\hline & $0-1$ & $2-3$ & $0-1$ & $2-3$ & $0-1$ & $2-3$ & $0-1$ & $2-3$ \\
\hline Median OS (month) & 5 & 47 & 26 & 28 & 11 & 33 & 8 & 33 \\
\hline 3 years OS (\%) & 0 & 52 & 45 & 48 & 38 & 51 & 30 & 51 \\
\hline
\end{tabular}

Abbreviations: OS, overall survival; TIE, tunica internal endothelial cell kinase.

Table 3 Angiopoietin and TIE expression in patient and tumor subsets

\begin{tabular}{|c|c|c|c|c|c|c|c|c|c|c|c|c|}
\hline & \multicolumn{3}{|c|}{ Angiopoietin-1 } & \multicolumn{3}{|c|}{ Angiopoietin-2 } & \multicolumn{3}{|c|}{ TIE1 } & \multicolumn{3}{|c|}{ TEK (TIE2) } \\
\hline & $0-1$ & $2-3$ & $P$ & $0-1$ & $2-3$ & $P$ & $0-1$ & $2-3$ & $P$ & $0-1$ & $2-3$ & $P$ \\
\hline \multicolumn{13}{|l|}{ Age } \\
\hline$\leqslant 67$ years & 3 & 26 & 0.45 & 15 & 13 & 0.57 & 8 & 21 & 0.55 & 5 & 24 & 0.73 \\
\hline$>67$ years & 4 & 18 & & 14 & 8 & & 8 & 14 & & 5 & 17 & \\
\hline \multicolumn{13}{|l|}{ Gender } \\
\hline Male & 3 & 18 & 1.0 & 13 & 8 & 0.77 & 7 & 14 & 1.0 & 6 & 15 & 0.28 \\
\hline Female & 4 & 26 & & 16 & 13 & & 9 & 21 & & 4 & 26 & \\
\hline \multicolumn{13}{|l|}{ Stage at diagnosis } \\
\hline Localized & 5 & 30 & 1.0 & 19 & 15 & 0.76 & 8 & 27 & 0.10 & 6 & 29 & 0.71 \\
\hline Metastatic & 2 & 14 & & 10 & 6 & & 8 & 8 & & 4 & 12 & \\
\hline \multicolumn{13}{|l|}{ Primary Site } \\
\hline Visceral/deep soft tissue & 5 & 15 & 0.29 & 13 & 7 & 0.15 & 10 & 10 & 0.14 & 5 & 15 & 0.45 \\
\hline HN/cutaneous & 1 & 19 & & 13 & 7 & & 5 & 15 & & 5 & 15 & \\
\hline Breast & 1 & 7 & & 3 & 4 & & 1 & 7 & & 0 & 8 & \\
\hline Stewart-Treves & 0 & 3 & & 0 & 3 & & 0 & 3 & & 0 & 3 & \\
\hline \multicolumn{13}{|l|}{ Radiation associated } \\
\hline Yes & 2 & 10 & 0.66 & 6 & 5 & 1.0 & 3 & 9 & 0.73 & 2 & 10 & 1.0 \\
\hline No & 5 & 34 & & 23 & 16 & & 13 & 26 & & 8 & 31 & \\
\hline \multicolumn{13}{|l|}{ Tumor size } \\
\hline$\leqslant 5 \mathrm{~cm}$ & 5 & 25 & 0.69 & 19 & 10 & 0.25 & 8 & 22 & 0.54 & 4 & 26 & 0.28 \\
\hline$>5 \mathrm{~cm}$ & 2 & 19 & & 10 & 11 & & 8 & 13 & & 6 & 15 & \\
\hline \multicolumn{13}{|l|}{ Histological pattern } \\
\hline Vasoformative & 2 & 28 & 0.11 & 13 & 16 & 0.04 & 3 & 27 & 0.0001 & 2 & 28 & 0.01 \\
\hline Non-vasoformative & 5 & 16 & & 16 & 5 & & 13 & 8 & & 8 & 13 & \\
\hline \multicolumn{13}{|l|}{ Necrosis } \\
\hline Present & 5 & 17 & 0.22 & 12 & 10 & 0.78 & 10 & 12 & 0.07 & 6 & 16 & 0.30 \\
\hline Absent & 2 & 27 & & 17 & 11 & & 6 & 23 & & 4 & 25 & \\
\hline
\end{tabular}

Abbreviation: TIE, tunica internal endothelial cell kinase.

Bold values highlight statistically significant differences.

in an array of malignancies. ${ }^{41-48}$ Importantly, agents targeting the ANGPT-TIE system are in active clinical development. ${ }^{49-52}$

Despite considerable examination of the roles of the ANGPT-TIE system in solid tumors, little information is available regarding the significance of this predominantly endothelium-limited signaling pathway in angiosarcoma. In our series of 51 angiosarcoma patients, we observed moderateto-strong angiopoietin-1 immunoreactivity in $86 \%$ of cases. Angiopoietin-1 is not expressed in cultured human endothelial cells nor in the vasculature of developing mouse embryos. ${ }^{25,53,54}$ Rather, angiopoietin-1 is thought to be expressed primarily by mural cells and signal through TEK (TIE2) in a paracrine fashion. ${ }^{25}$ Thus, our observations raise the possibility that, in contrast to cultured and fetal endothelial cells, angiosarcoma cells express angiopoietin-1. This is consistent with the findings of Brown et $\mathrm{al}^{28}$ who detected angiopoietin-1 mRNA in two of two angiosarcomas studied. Alternatively, angiopoietin-1 immunoreactivity may be attributable to detection of receptor-bound angiopoietin-1. Consistent with this possibility is the observation that angiopoietin-1 protein is observed in the vessels of unwounded skin. ${ }^{55}$ Our observation that four of seven angiosarcomas with no-to-weak angiopoietin-1 staining demonstrated 
moderate-to-strong TEK (TIE2) expression suggests that detection of bound angiopoietin-1 does not entirely explain the near universal angiopoietin-1 immunoreactivity seen in angiosarcomas. Further investigation of the cellular source of angiopoietin-1 in angiosarcomas is warranted.

Our observation that $62 \%$ of angiosarcomas express at least low levels of angiopoietin-2 is consistent with the endothelial differentiation of this malignancy and the scant available data regarding angiopoietin-2 expression in angiosarcoma. Brown et $a l^{28}$ detected strong angiopoietin-2 mRNA expression in two of two examined angiosarcomas. Angiopoietin-2 mRNA was also found to be upregulated in angiosarcomas when compared with other soft tissue sarcomas. ${ }^{15}$ Amo et $a l^{53}$ observed elevated serum levels of angiopoietin-2 in 11 patients with pathologically proven angiosarcoma of the face and scalp compared with healthy volunteers. ${ }^{53}$ In this study, serum angiopoietin-2 was found to correlate with disease burden. The high rates of TIE1 and TEK (TIE2) expression in our patient cohort are also consistent with endothelial differentiation and the limited evidence of TIE receptor expression in angiosarcoma. Strong TIE1 and TEK (TIE2) mRNA expression has been observed in two of two angiosarcomas. ${ }^{28}$ Furthermore, levels of TIE1 and TEK (TIE2) mRNA were found to be elevated in angiosarcomas compared with other soft tissue sarcomas. ${ }^{15}$ In addition, TEK (TIE2) expression was identified immunohistochemically in 7 of 10 examined pulmonary epithelioid angiosarcomas. ${ }^{12}$

Our observation that increased expression of ANGPT-TIE system components is associated with both the more favorable vasoformative histological pattern and improved overall survival is consistent with a loss of endothelial markers with progression to more aggressive disease. ${ }^{30}$ Consistent with this model is the observation that loss of vascular endothelial growth factor receptor 2 is associated with worse survival in angiosarcoma patients. ${ }^{9}$ The correlation between increased angiopoietin-1 expression and overall survival is not simply explained by correlations with other known prognostic factors. The dominant, negative prognostic factor in this patient population is the presence of metastatic disease at diagnosis. The fact that angiopoietin-1 expression did not vary when patients were segregated by the presence or absence of metastases at diagnosis (nor any other examined clinical or pathological variable) suggests it may be a novel prognostic factor in this disease. Larger studies are required to confirm this observation and define the potential utility of using angiopoietin-1 immunoreactivity in clinical testing.

TEK (TIE2) inhibitor therapy has recently been reported to inhibit angiosarcoma growth in two distinct murine models of this disease. ${ }^{29}$ Coupled with our observation that ANGPT-TIE system components are commonly expressed in angiosarcoma, these findings raise the possibility that manipulation of the ANGPT-TIE system may have therapeutic utility in this poor prognosis malignancy. Thus, immunohistochemical evaluation of these proteins in angiosarcoma could potentially be used as a part of therapeutic decision making. Further studies on the mechanistic roles for this critical vascular signaling pathway in angiosarcomagenesis are certainly merited.

\section{Acknowledgements}

This work was supported by NIH grant 1UL1 RR025011 (KRK).

\section{Disclosure/conflict of interest}

The authors declare no conflict of interest.

\section{References}

1 Abraham JA, Hornicek FJ, Kaufman AM, et al. Treatment and outcome of 82 patients with angiosarcoma. Ann Surg Oncol 2007;14:1953-1967.

2 Fayette J, Martin E, Piperno-Neumann S, et al. Angiosarcomas, a heterogeneous group of sarcomas with specific behavior depending on primary site: a retrospective study of 161 cases. Ann Oncol 2007;18:2030-2036.

3 Fury MG, Antonescu CR, Van Zee KJ, et al. A 14-year retrospective review of angiosarcoma: Clinical characteristics, prognostic factors, and treatment outcomes with surgery and chemotherapy. Cancer J 2005;11: 241-247.

4 Mark RJ, Poen JC, Tran LM, et al. Angiosarcoma. A report of 67 patients and a review of the literature. Cancer 1996;77:2400-2406.

5 Naka N, Ohsawa M, Tomita Y, et al. Prognostic factors in angiosarcoma: a multivariate analysis of 55 cases. J Surg Oncol 1996;61:170-176.

6 Penel N, Marreaud S, Robin YM, et al. Angiosarcoma: state of the art and perspectives. Crit Rev Oncol Hematol 2011;80:257-263.

7 Brown LF, Tognazzi K, Dvorak HF, et al. Strong expression of kinase insert domain-containing receptor, a vascular permeability factor/vascular endothelial growth factor receptor in AIDS-associated Kaposi's sarcoma and cutaneous angiosarcoma. Am J Pathol 1996;148:1065-1074.

8 Hashimoto M, Ohsawa M, Ohnishi A, et al. Expression of vascular endothelial growth factor and its receptor mRNA in angiosarcoma. Lab Invest 1995;73:859-863.

9 Itakura E, Yamamoto H, Oda Y, et al. Detection and characterization of vascular endothelial growth factors and their receptors in a series of angiosarcomas. J Surg Oncol 2008;97:74-81.

10 Lahat G, Dhuka AR, Hallevi H, et al. Angiosarcoma: clinical and molecular insights. Ann Surg 2010;251: 1098-1106.

11 Ohsawa M, Naka N, Tomita Y, et al. Use of immunohistochemical procedures in diagnosing angiosarcoma. Evaluation of 98 cases. Cancer 1995;75:2867-2874. 
12 Stacher E, Gruber-Mosenbacher U, Halbwedl I, et al. The VEGF-system in primary pulmonary angiosarcomas and haemangioendotheliomas: new potential therapeutic targets? Lung Cancer 2009;65:49-55.

13 Tokuyama W, Mikami T, Masuzawa M, et al. Autocrine and paracrine roles of VEGF/VEGFR-2 and VEGF-C/ VEGFR-3 signaling in angiosarcomas of the scalp and face. Hum Pathol 2010;41:407-414.

14 Zietz C, Rossle M, Haas C, et al. MDM-2 oncoprotein overexpression, p53 gene mutation, and VEGF up-regulation in angiosarcomas. Am J Pathol 1998;153: 1425-1433.

15 Antonescu CR, Yoshida A, Guo T, et al. KDR activating mutations in human angiosarcomas are sensitive to specific kinase inhibitors. Cancer Res 2009;69: 7175-7179.

16 Maki RG, D’Adamo DR, Keohan ML, et al. Phase II study of sorafenib in patients with metastatic or recurrent sarcomas. J Clin Oncol 2009;27:3133-3140.

17 Ray-Coquard I, Italiano A, Bompas E, et al. Sorafenib for patients with advanced angiosarcoma: a phase II Trial from the French Sarcoma Group (GSF/GETO). Oncologist 2012;17:260-266.

18 Agulnik M, Yarber JL, Okuno SH, et al. An open-label, multicenter phase II study of bevacizumab for the treatment of angiosarcoma and epithelioid hemangioendotheliomas. Ann Oncol 2013;24:257-263.

19 Rosen A, Thimon S, Ternant D, et al. Partial response to bevacizumab of an extensive cutaneous angiosarcoma of the face. Br J Dermatol 2010;163:225-227.

20 Huang $\mathrm{H}$, Bhat $\mathrm{A}$, Woodnutt $\mathrm{G}$, et al. Targeting the ANGPT-TIE2 pathway in malignancy. Nat Rev Cancer 2010;10:575-585.

21 Fukuhara S, Sako K, Minami T, et al. Differential function of Tie2 at cell-cell contacts and cell-substratum contacts regulated by angiopoietin-1. Nat Cell Biol 2008;10:513-526.

22 Fukuhara S, Sako K, Noda K, et al. Tie2 is tied at the cell-cell contacts and to extracellular matrix by angiopoietin-1. Exp Mol Med 2009;41:133-139.

23 Kanda S, Miyata Y, Mochizuki Y, et al. Angiopoietin 1 is mitogenic for cultured endothelial cells. Cancer Res 2005;65:6820-6827.

24 Saharinen P, Eklund L, Miettinen J, et al. Angiopoietins assemble distinct Tie2 signalling complexes in endothelial cell-cell and cell-matrix contacts. Nat Cell Biol 2008;10:527-537.

25 Kim I, Kim HG, So JN, et al. Angiopoietin-1 regulates endothelial cell survival through the phosphatidylinositol 3'-Kinase/Akt signal transduction pathway. Circ Res 2000;86:24-29.

$26 \mathrm{Hu} \mathrm{B}$, Cheng SY. Angiopoietin-2: development of inhibitors for cancer therapy. Curr Oncol Rep 2009;11:111-116.

27 Valenzuela DM, Griffiths JA, Rojas J, et al. Angiopoietins 3 and 4: diverging gene counterparts in mice and humans. Proc Natl Acad Sci USA 1999;96:1904-1909.

28 Brown LF, Dezube BJ, Tognazzi K, et al. Expression of Tie1, Tie2, and angiopoietins 1, 2, and 4 in Kaposi's sarcoma and cutaneous angiosarcoma. Am J Pathol 2000;156:2179-2183.

29 Hasenstein JR, Kasmerchak K, Buehler D, et al. Efficacy of Tie2 receptor antagonism in angiosarcoma. Neoplasia 2012;14:131-140.

30 Shon W, Jenkins SM, Ross DT, et al. Angiosarcoma: a study of 98 cases with immunohistochemical evaluation of TLE3, a recently described marker of potential taxane responsiveness. J Cutan Pathol 2011;38: 961-966.

31 Maisonpierre PC, Suri C, Jones PF, et al. Angiopoietin2, a natural antagonist for Tie2 that disrupts in vivo angiogenesis. Science 1997;277:55-60.

32 Suri C, Jones PF, Patan S, et al. Requisite role of angiopoietin-1, a ligand for the TIE2 receptor, during embryonic angiogenesis. Cell 1996;87:1171-1180.

33 Gale NW, Thurston G, Hackett SF, et al. Angiopoietin-2 is required for postnatal angiogenesis and lymphatic patterning, and only the latter role is rescued by Angiopoietin-1. Dev Cell 2002;3:411-423.

34 Dumont DJ, Gradwohl G, Fong GH, et al. Dominantnegative and targeted null mutations in the endothelial receptor tyrosine kinase, tek, reveal a critical role in vasculogenesis of the embryo. Genes Dev 1994;8: 1897-1909.

35 Sato TN, Tozawa Y, Deutsch U, et al. Distinct roles of the receptor tyrosine kinases Tie-1 and Tie-2 in blood vessel formation. Nature 1995;376:70-74.

36 Puri MC, Rossant J, Alitalo K, et al. The receptor tyrosine kinase TIE is required for integrity and survival of vascular endothelial cells. EMBO J 1995;14:5884-5891.

37 Chung YC, Hou YC, Chang CN, et al. Expression and prognostic significance of angiopoietin in colorectal carcinoma. J Surg Oncol 2006;94:631-638.

38 Dhiwakar M, Malone JP, Kay PA, et al. Use of angiopoietin-1 expression in squamous cell carcinoma of the head and neck to predict disease-free survival. 2010 ASCO Annual Meeting 2010;28:abstr 5542.

39 Oka N, Yamamoto Y, Takahashi M, et al. Expression of angiopoietin-1 and -2, and its clinical significance in human bladder cancer. BJU Int 2005;95:660-663.

40 Tanaka F, Ishikawa S, Yanagihara K, et al. Expression of angiopoietins and its clinical significance in non-small cell lung cancer. Cancer Res 2002;62: 7124-7129.

41 Sfiligoi C, de Luca A, Cascone I, et al. Angiopoietin-2 expression in breast cancer correlates with lymph node invasion and short survival. Int J Cancer 2003;103:466-474.

42 Mitsuhashi N, Shimizu H, Ohtsuka M, et al. Angiopoietins and Tie-2 expression in angiogenesis and proliferation of human hepatocellular carcinoma. Hepatology 2003;37:1105-1113.

43 Ahmad SA, Liu W, Jung YD, et al. The effects of angiopoietin-1 and -2 on tumor growth and angiogenesis in human colon cancer. Cancer Res 2001;61:1255-1259.

44 Srirajaskanthan R, Dancey G, Hackshaw A, et al. Circulating angiopoietin-2 is elevated in patients with neuroendocrine tumours and correlates with disease burden and prognosis. Endocr Relat Cancer 2009;16:967-976.

45 Wong MP, Chan SY, Fu KH, et al. The angiopoietins, tie2 and vascular endothelial growth factor are differentially expressed in the transformation of normal lung to non-small cell lung carcinomas. Lung Cancer 2000;29:11-22.

46 Lind AJ, Wikstrom P, Granfors T, et al. Angiopoietin 2 expression is related to histological grade, vascular density, metastases, and outcome in prostate cancer. Prostate 2005;62:394-399.

47 Hata K, Nakayama K, Fujiwaki R, et al. Expression of the angopoietin-1, angopoietin-2, Tie2, and vascular endothelial growth factor gene in epithelial ovarian cancer. Gynecol Oncol 2004;93:215-222. 
48 Schulz P, Fischer C, Detjen KM, et al. Angiopoietin-2 drives lymphatic metastasis of pancreatic cancer. FASEB J 2011;25:3325-3335.

49 Herbst RS, Hong D, Chap L, et al. Safety, pharmacokinetics, and antitumor activity of AMG 386, a selective angiopoietin inhibitor, in adult patients with advanced solid tumors. J Clin Oncol 2009;27:3557-3565.

50 Karlan BY, Oza AM, Richardson GE, et al. Randomized, double-blind, placebo-controlled phase II study of AMG 386 combined with weekly paclitaxel in patients with recurrent ovarian cancer. J Clin Oncol 2012;30:362-371.

51 Mita AC, Takimoto CH, Mita M, et al. Phase 1 study of AMG 386, a selective angiopoietin 1/2-neutralizing peptibody, in combination with chemotherapy in adults with advanced solid tumors. Clin Cancer Res 2010;16:3044-3056.
52 Rini B, Szczylik C, Tannir NM, et al. AMG 386 in combination with sorafenib in patients with metastatic clear cell carcinoma of the kidney: A randomized, double-blind, placebocontrolled, phase 2 study. Cancer 2012;118: 6152-6161.

53 Amo Y, Masuzawa M, Hamada Y, et al. Observations on angiopoietin 2 in patients with angiosarcoma. Br J Dermatol 2004;150:1028-1029.

54 Davis S, Aldrich TH, Jones PF, et al. Isolation of angiopoietin-1, a ligand for the TIE2 receptor, by secretion-trap expression cloning. Cell 1996;87: 1161-1169.

55 Staton CA, Valluru M, Hoh L, et al. Angiopoietin-1, angiopoietin-2 and Tie-2 receptor expression in human dermal wound repair and scarring. $\mathrm{Br} \mathrm{J}$ Dermatol 2010;163:920-927. 